\title{
Tissue oximetry in anaesthesia and intensive care
}

\author{
Aleksandra Biedrzycka ${ }^{1}$, Romuald Lango ${ }^{2}$ \\ ${ }^{1}$ Department of Anaesthesiology and Intensive Therapy, University Clinical Centre in Gdańsk, Poland \\ ${ }^{2}$ Department of Cardiac Anaesthesiology, Medical University of Gdańsk, Poland
}

\begin{abstract}
Conventional monitoring during surgery and intensive care is not sufficiently sensitive to detect acute changes in vital organs perfusion, while its good quality is critical for maintaining their function. Disturbed vital organ perfusion may lead to the development of postoperative complications, including neurological sequel and renal failure. Nearinfra-red spectroscopy (NIRS) represents one of up-to-date techniques of patient monitoring which is commonly used for the assessment of brain oximetry in thoracic aorta surgery, and - increasingly more often -in open-heart surgery. Algorithms for maintaining adequate brain saturation may result in a decrease of neurological complications and cognitive dysfunction following cardiac surgery. The assessment of kidney and visceral perfusion with tissue oximetry is gaining increasing interest during pediatric cardiac surgery. Attempts at decreasing complications by the use of brain oximetry during carotid endarterectomy, as well as thoracic and abdominal surgery demonstrated conflicting results. In recent years NIRS technique was proposed as a tool for muscle perfusion assessment under short term ischemia and reperfusion, referred to as vascular occlusion test (VOT). This monitoring extension allows for the identification of early disturbances in tissue perfusion. Results of recent studies utilizing VOT suggest that the muscle saturation decrease rate is reduced in septic shock patients, while decreased speed of saturation recovery on reperfusion is related to disturbed microcirculation. Being non-invasive and feasible technique, NIRS offers an improvement of preoperative risk assessment in cardiac surgery and promises more comprehensive intraoperative and ICU patient monitoring allowing for better outcome.
\end{abstract}

Key words: tissue oximetry, brain saturation, stroke

Anaesthesiology Intensive Therapy 2016, vol. 48, no 1, 41-48

Surgical procedures are performed in an increasingly elderly population of patients with numerous concomitant diseases and severe general health status who have to be treated in intensive care units (ICUs); therefore, early diagnosis and prediction of disorders likely to deteriorate their conditions and lead to death are crucial. In order to find the correlation between impaired perfusion of organs, such as the brain, kidneys, gastrointestinal tract (Gl tract), and their sequels, e.g. neurocognitive disorders, acute renal injury and multiple organ dysfunction syndrome, new monitoring techniques are required to enable early identification of risks and implementation of therapeutic interventions. The major goal of preoperative optimization of cardiovascular function is to maintain an adequate blood flow through the vital organs, such as the brain, heart, kidneys, liver, and Gl walls. For practical reasons, the most commonly monitored haemodynamic parameter is arterial pressure. However, cardiac output and appropriate distribution of blood flow through the individual organs can be of greater importance for maintaining organ function and structure. The haemodynamic monitoring techniques currently routinely used in operating theatres and ICUs do not allow to assess microcirculation and distribution of blood flow to organs or to detect early stages of cardiovascular dysfunctions when the compensatory mechanisms activated are capable of maintaining proper arterial pressure.

Numerous recent literature reports have been focused on near-infra-red spectroscopy (NIRS), which involves measurements of haemoglobin oxygen saturation in blood flowing through a particular organ. The study findings demonstrate that this dynamically developing monitoring technique, predominantly used during cardiac and thoracic 
aorta surgeries to assess brain saturation, can be a major step forward in perioperative assessment of patient's health status.

\section{PHYSICAL AND BIOLOGICAL BASIS OF NEAR-INFRA-RED SPECTROSCOPY}

Near-infra-red spectrometry, first described nearly 40 years ago [1], relies on measurements of the intensity of radiation at a $730-910 \mathrm{~nm}$ wavelength, depending on the type of monitoring device. The radiation emitted by a diode or laser undergoes reflection, deflection, dispersion and absorption. The relationship between absorbance of radiation passing through the tissue and concentration of a substance is described by the Lambert-Beer law. The absorption of a part of photons of emitted light by chromophores, mainly oxyhaemoglobin and deoxyhaemoglobin but also myoglobin and cytochrome $C$ oxidase, enables to assess their concentrations [2]. The extent of chromophore oxygenation affects the absorption of light travelling through the tissue, which allows to evaluate its oxygenation. Unlike pulse oximetry, the NIRS technique does not enable to differentiate arterial and venous blood. Hence, the measurements concern mixed blood. The major clinical application of NIRS is measurement of brain saturation. Since the venous blood is responsible for about $70 \%$ of absorption of radiation in the cerebral cortex while the arterial blood for $25 \%$ and capillary blood for $5 \%$, a decrease in brain saturation refers mainly to venous blood, which predominantly results from a local increase in oxygen extraction [3].

A sensor used for tissue saturation measurements, also called an optode, consists of the source of light and two detectors. Depending on type of device, the source of light is a light-emitting diode (LED) (INVOS and Equanox) or a laser (Fore-Sight, NIRO-300 and CerOx), emitting electromagnetic radiation in the near-infra-red range. The photon path between the source of light and the detector is elliptic and reaches the depth of about $1 / 3$ of the distance between them. In order to eliminate the interferences resulting from radiation absorption in the penetrated structures between the tissue examined and the sensor, two detectors are used placed at a close distance [4]. The closer one records measurements from shallower layers, i.e. the skin, epicranial aponeurosis, cranium and meninges. The value measured by this sensor is subtracted from the value recorded by the farther sensor, which allows to estimate blood saturation in the brain. It is estimated that about $85 \%$ of a photon beam permeates through the cortex whereas 15\% through more superficial structures [5]. Manufacturers of the devices in question apply various distances between the source of light and receivers, e.g. 30 and $40 \mathrm{~mm}$ in INVOS, 15 and $50 \mathrm{~mm}$ in Fore-Sight. In the INVOS monitor, the depth of penetration is about $1.7 \mathrm{~cm}$.
Sensors placed each on either side at the level of $2-3 \mathrm{~cm}$ above the orbit border allow to monitor oxygenation of the cerebral cortex supplied by the anterior and medial cerebral artery. In order to interpret properly the monitor recordings of brain oximetry, especially in devices based on LEDs, it is essential to determine the baseline value, i.e. brain saturation before anaesthesia induction in patients who breathe ambient air. Measurements performed during the procedure are compared with this value. An intraoperative decrease in brain saturation by more than $20 \%$ [1] or $25 \%$ [6] of the baseline value [2] and a decrease in absolute value of saturation below $50 \%$ are associated with postoperative cognitive dysfunctions $[2,7]$ as well as a higher risk of stroke or coma [8].

Monitoring of brain saturation based on NIRS has many assets, including non-invasiveness, simple interpretation of results and their real-time presentation, which enables early interventions and increases the safety of anaesthesia. The accuracy of measurements is not affected by the anaesthetic chosen and the use of electrocoagulation. The measurement of tissue saturation is a sensitive method of monitoring the quality of brain protection during total circulatory arrest in deep hypothermia, when other methods, including analysis of EEG signals, are found difficult to interpret.

\section{LIMITATIONS OF NIRS FOR ASSESSMENT OF BRAIN SATURATION}

As any other monitoring technique, brain oximetry is not free of weak points. The factors potentially impairing NIRS recordings include the amount of skin pigment and a high concentration of conjugated bilirubin. The signal detection can also be disturbed by a strong external source of light in the operating theatre or reduced adhesion of electrodes due to e.g. increased perspiration of patients. Moreover, an epior a subdural haematoma can have adverse effects; in such cases, the cerebral cortex is likely to be beyond the range of radiation emitted by the sensor. The other factors limiting the value of brain saturation measurements involve brain oedema and local disorders of perfusion resulting from the presence of atherosclerotic lesions or arterial embolism [9].

In cases of cerebral cortex atrophy, often observed in elderly patients, the range of proton beam can be insufficient to assess cortex oxygenation (Fig.2)[9]. It has been found that NIRS monitor readings in the elderly can paradoxically decrease during cooling and increase during re-warming (brain cooling reactivity), which indicates the presence of significant cortical atrophies and limits the possibility of brain oximetry interpretation [5]. Moreover, oximetry recordings can be hindered by vasoconstrictors [10]. Noradrenaline-induced vasoconstriction of skin vessels has been observed to have markedly stronger effects on brain oximeter recordings than a decrease in cardiac 


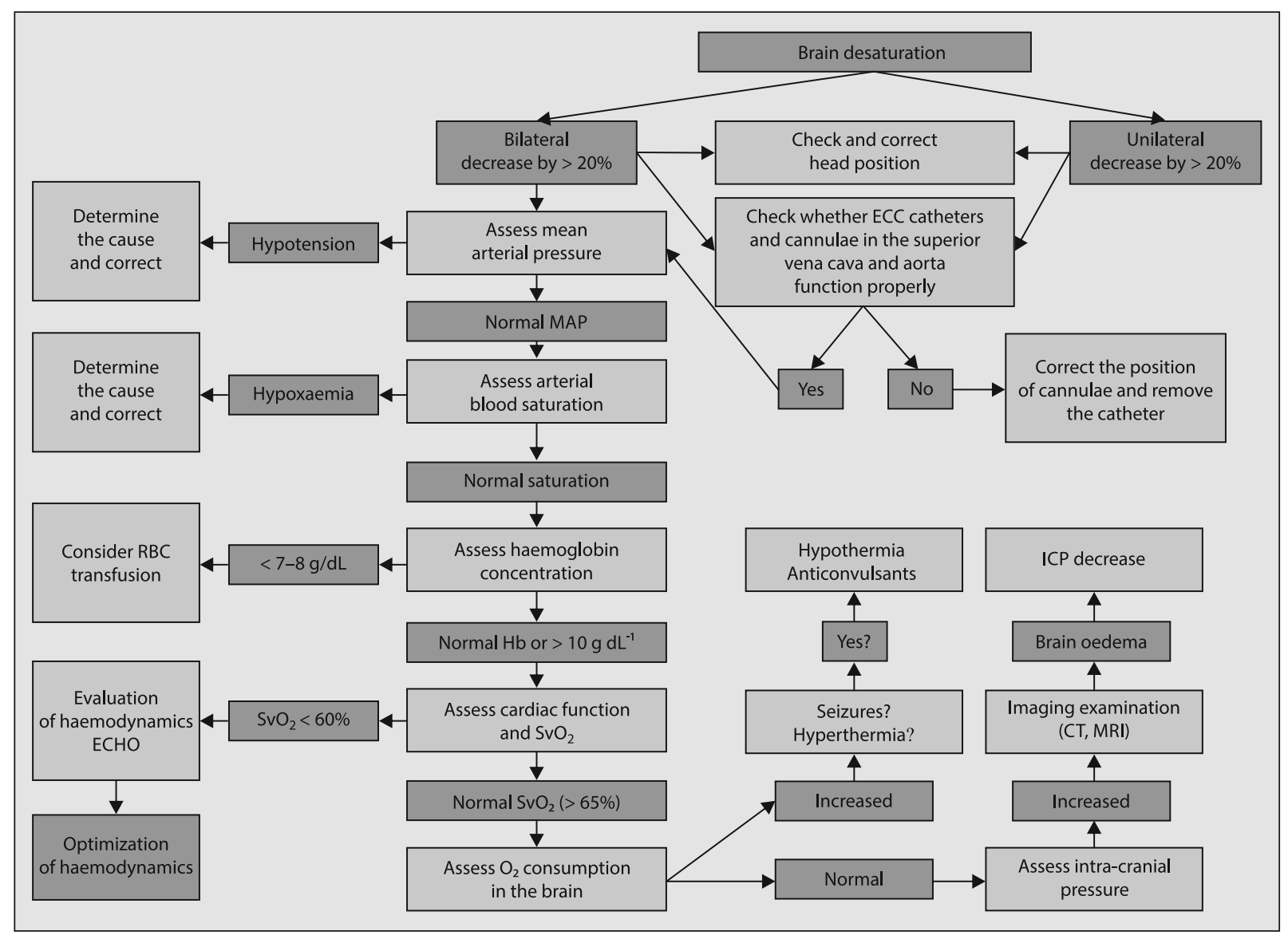

Figure 1. Algorithm of management for brain saturation decreases during cardiac surgery [12]

output and $\mathrm{CO} 2$ pressure changes in the blood [9]. Grocott and colleagues [11] have demonstrated that changes in blood oxygenation in the head skin can be responsible for $17 \%$ of saturation changes recorded by the NIRS method. The monitor can show no changes in tissue saturation when the transport of oxygen to the cerebral cortex and its consumption change equally. The difficulties in the assessment of effectiveness of interventions aimed at restoration of proper brain saturation are caused by differences in the values measured with individual types of devices. While measurements of absolute values of brain saturation are affected by the limitations described above, brain saturation trends allow to interpret properly the effects of various factors on brain oxygenation [12].

\section{USE OF BRAIN OXIMETRY DURING CARDIAC SURGERY}

Since the highest risk of appearance of factors impairing transport of oxygen to the brain is associated with extracorporeal circulation, brain saturation is most commonly monitored during cardiac surgical procedures. Insufficient transport of oxygen compared to brain requirements can be caused by both decreased arterial pressure or haemodilution and increased consumption of oxygen during re-warming before the weaning from extracorporeal circulation. The factors likely to reduce brain saturation include reduced oxygenation of arterial blood, hypocapnia and the resultant vasoconstriction of cerebral arteries, impaired flow through the venous cannula of extracorporeal circulation as well as micro- and macro-embolism and non-pulsatile blood flow $[13,14]$. The above factors increase the risk of neurological complications following cardiac surgical procedures, compared to other surgical fields [10].

Monitoring of brain saturation and interventions to restore its proper values are likely to improve treatment outcomes, particularly the incidence of neurological complications and postoperative cognitive dysfunctions [10]. During thoracic aorta dissection surgeries monitoring of brain oximetry frequently indicate the need to change the way of cannulation for selective cerebral perfusion. Numerous case reports reveal that brain saturation monitoring during such procedures enables to diagnose brain hypoxia, which is not implied by other routinely monitored parameters [10]. 


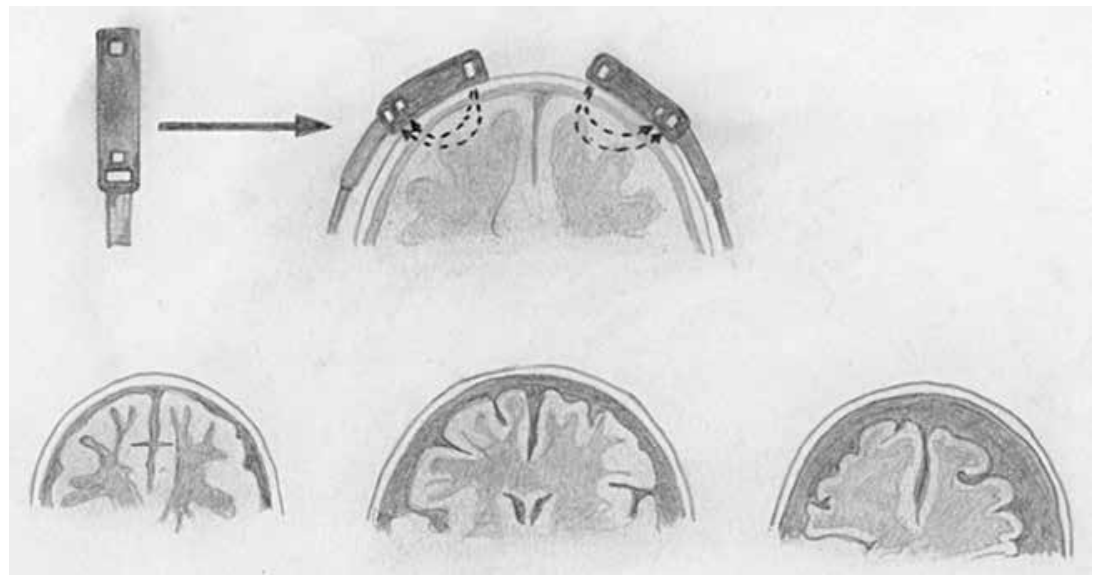

Figure 2. Schematic presentation of the range of a light beam during measurements of brain saturation. Bottom drawings - anatomical relationships in a child, adult and elderly individual with cerebral cortex atrophy, in whom the range of light beam can be insufficient to measure brain saturation (by Paweł Żelechowski, MD, PhD)

A decrease in brain saturation below the borderline values mentioned earlier during cardiac or thoracic aorta surgery indicates that transport of oxygen to the brain is insufficient to meet its metabolic requirements. The algorithm of management in cases of reduced brain saturation is presented in Fig.1. Its use was found effective in $88 \%$ of cases of reduced saturation; however, its individual elements showed different effectiveness - from no effect of head positioning to $100 \%$ effectiveness in response to the corrected position of the venous cannula of extracorporeal circulation and after red-cell concentrate transfusion [15].

Cardiac surgery patients with episodes of reduced brain saturation are at a higher risk of complications, such as respiratory failure, myocardial infarction, reoperation and postoperative cognitive dysfunctions [7].

The use of brain saturation as a baseline parameter for decision-making about interventions to increase the transport of oxygen can adversely affect the CNS condition and the function of other organs. The management aimed at maintenance of proper brain saturation during cardiac surgery has been found to reduce the risk of stroke and to be associated with lower incidences of multiple organ failure. An intraoperative reduction in brain saturation can also be correlated with prolonged treatment in ICUs and increased mortality [16].

The studies in patients undergoing off-pump coronary artery bypass grafting (OPCABG) reveal that reduced brain saturation precedes a decrease in saturation of mixed venous blood [17]. Therefore, a hypothesis has been put forward that early introduction of management to restore proper brain oxygenation can also contribute to quick improvement in oxygen balance.

It is currently believed that brain saturation measured directly immediately before cardiac surgery is of a prognostic value. Its low value has been observed to correlate with a high risk of surgery according to the EuroSCORE as well as poor diastolic and systolic functions of the left ventricle and to indicate an increased risk of postoperative delirium [17, 18]. Heringlake and co-workers [19] have demonstrated the lowest value of brain saturation immediately prior to cardiac surgery, i.e. below $50 \%$, is associated with increased 30 -day and annual mortality.

The studies conducted to date showing benefits of brain saturation monitoring during cardiac surgery involved small populations of patients and varied in methods and devices used, which hinders the comparison of findings [20]. The review of 43 publications enclosing over 50 thousand adult cardiac surgery patients did not explicitly confirm the benefits resulting from interventions undertaken based on NIRS recordings, thus further studies are required [20]. In search of a better parameter related to the risk of neurological complications, determinations of the extent and duration of reduced brain saturation, as a desaturation load, have been suggested [15]. The randomized clinical trial has demonstrated the effectiveness of interventions administered according to the defined protocol in order to reduce the desaturation load; however, as suggested by the authors, further studies are needed to confirm the correlation between decreased desaturation load and improved treatment outcomes [15].

In Poland, the pioneer studies on brain saturation monitoring come from the Silesian Centre for Heart Diseases in Zabrze. Kucewicz and colleagues [21] proposed a clear-cut management algorithm in cases with brain saturation reduced by $30 \%$ as compared to the baseline value or $<50 \%$ of the absolute value. The algorithm takes into account a noradrenaline-induced increase in arterial pressure, an increase in cardiac output, improvement of venous flow, possible repositioning of cannulae, deepening of anaesthesia to reduce brain oxygen requirements, an increase in oxygen content in the respiratory mixture, correction of 
ventilation to maintain proper pressure of carbon dioxide or possible institution of nitroglycerine infusion, and others.

\section{USE OF BRAIN OXIMETRY IN OTHER SURGICAL FIELDS}

Carotid endarterectomy is associated with a high risk of cerebral stroke. It has been postulated that brain saturation monitoring during such procedures could reduce the risk of critical cerebral ischaemia on the side of the artery operated on $[22,24]$. Although transcranial Doppler (TCD), consisting in monitoring of a decrease in blood flow rate in the middle cerebral artery through the temporal acoustic window, can provide more precise identification of patients at risk of ischaemia [25], its use in the operating theatre is technically difficult and troublesome; moreover, in about $20 \%$ of individuals TCD is infeasible [22]. The sensitivity of brain saturation measurements to detect significantly reduced cerebral flows is comparable with TCD yet its specificity is low, thus can lead to false positive results. Therefore, decisions about temporary shunts based on brain saturation measurements could lead to their use in many patients in whom they are not necessary [25]. Nevertheless, Mille and co-workers [26] have demonstrated that if brain saturation does not decrease by more than $20 \%$ within the first 2 minutes after carotid artery clamping, ischaemic stroke is highly unlikely and the shunt should not be required. According to another study, a decrease in brain saturation by even 13\% measured using NIRO-300 (Hamamatsu Photonics, Japan) is consistent with the EEG changes characteristic of cerebral ischaemia in $97 \%$ of cases [27].

Furthermore, studies concerning the use of brain saturation measurements to improve treatment outcomes were also conducted during thoracic surgical procedures with one-lung ventilation [28] and in elderly patients undergoing extensive abdominal procedures [6]. Tang and colleagues [29] have observed a relationship between low values of brain saturation and cognitive dysfunctions in the early postoperative period following the procedures with onelung ventilation. Moreover, patients with a decrease in brain saturation by at least $25 \%$ were hospitalized substantially longer [30]. Even short episodes of reduced brain saturation below $65 \%$, which were observed in more than half of patients, were associated with a two-fold higher incidence of consciousness disorders. Additionally, elderly patients with restored proper values of brain saturation showed lower incidences of cognitive dysfunctions and required shorter ICU treatment [30].

\section{ATTEMPTS TO USE OXIMETRY FOR EVALUATION OF OXYGENATION OF OTHER ORGANS}

The search for clinical applications of tissue saturation monitoring using NIRS is not limited to brain saturation.
The attempts have been made to evaluate tissue saturation of kidneys, intestines, liver as well as thoracic and brachial muscles. During cardiac surgical procedures in newborns, a spectrophotometric sensor is used to monitor tissue saturation in the kidney. Its low values have been found to be associated with increased concentration of creatinine after surgery and the risk of acute kidney injury [31]. Moreover, there were some attempts to use the oximeter to measure saturation of the liver, yet no clinical benefits have been observed [32]. In newborns undergoing surgeries due to congenital heart defects, splanchnic saturation, measured during weaning from extracorporeal circulation using the sensor placed directly below the navel, has been demonstrated to be the parameter, which enables more accurate prediction of increased requirements for vasoconstrictors and prolonged artificial lung ventilation, as compared to renal and cerebral saturations [33]. An interesting application of tissue oximetry in newborns was suggested by Fortune and colleagues [34], who emphasized the importance of the cerebro-splanchnic oxygenation ratio (CSOR). Under normal circumstances, the value of brain saturation is lower than that of splanchnic saturation due to higher oxygen extraction in the brain. A ratio increase above 1 indicates the risk of intestinal ischaemia, predominantly due to splanchnic vasoconstriction caused by circulation centralization.

\section{USE OF OXIMETRY FOR EVALUATION OF MICROCIRCULATION AND OXYGEN CONSUMPTION IN TISSUES}

The application of measurements of tissue saturation in the muscles to evaluate the consumption of oxygen in the tissue and reactivity of microcirculation during artificially induced short-term ischaemia followed by reperfusion was suggested by Gomez and co-workers [35]. For this purpose, the vascular occlusion test (VOT) is used to analyse the changes in muscle tissue saturation during and immediately after short-term closure of blood flow in the limb using a sphygmomanometer (Fig. 3).

During the test, the baseline value of muscle saturation is set, the cuff inflated above the systolic pressure and the inflow of blood closed. After the time planned, the cuff is deflated and the flow restored. As a result of transient hypoxia, during reperfusion arterioles dilate, capillary refill increases and reactive congestion is observed [36]. The VOT evaluates the rate of muscle saturation decreases after flow closure as well as the time between the cuff release and maximum saturation when non-oxygenated haemoglobin is washed out. It is implied that the rate of muscle saturation decreases is proportional to oxygen consumption while the rate of saturation increases after flow restoration is a marker of the efficacy of vasodilating mechanisms [35]. The oximetric sensor is placed over various sites, most commonly on the 


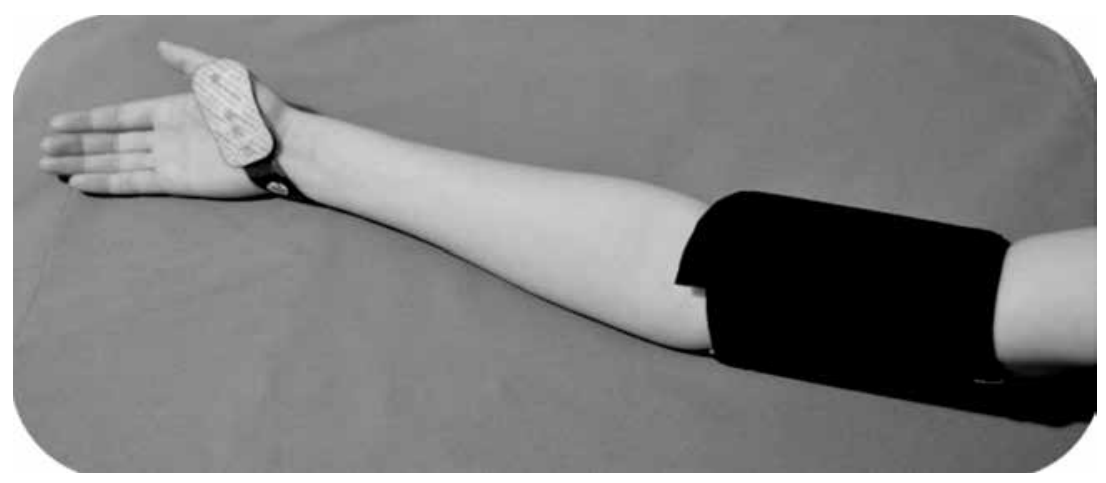

Figure 3. Preparation for the vascular occlusion test (VOT) in the upper limb

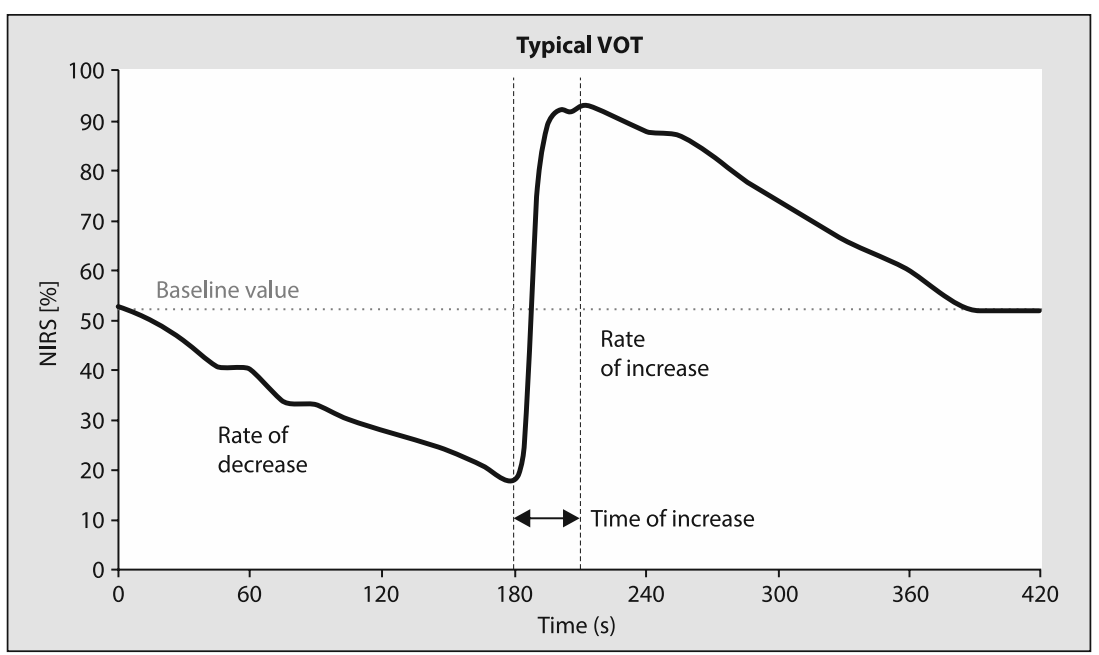

Figure 4. Typical course of tissue saturation changes during VOT

thenar, but also above the group of flexors on the forearm or calf muscles. The comparison of results is hindered by the use of various ischaemia thresholds, some based on the time of ischaemia, 3 or 5 minutes, and some on a defined value of tissue saturation at which the flow is restored. An example graph of tissue oximetry during VOT is presented in Fig.4.

In numerous recent studies, the VOT data were compared to the patient status parameters monitored routinely in intensive care. Lower baseline values of tissue saturation were found to be accompanied by higher concentrations of lactates, clinical symptoms of peripheral hypoperfusion, such as the difference in temperature between the forearm and finger bulb exceeding $4^{\circ} \mathrm{C}$, and prolonged time of capillary flow [37]. According to Lima and colleagues [38], in patients with lactic acidosis admitted to the ICU, the maintenance of reduced tissue saturation in the thenar muscles after fluid resuscitation was associated with a higher number of failed organs.
The attempts to use the VOT for clinical evaluation of microcirculation were initially made in patients with septic shock. The observation that their baseline values of tissue saturation in muscles are reduced $[39,40]$ has not been confirmed in other studies [41,42]. Pareznik and co-workers [42] have demonstrated that the rate of tissue saturation decreases during the flow arrest in the limb was lower in septic shock patients than in the remaining patients, which was explained by impaired abilities of oxygen extraction and its consumption in tissues. In survivors, the restoration of the rate of saturation decreases during the VOT was observed.

Some equally relevant data on microcirculation in patients with septic shock are provided by interpretation of the rate of tissue saturation increases after flow restoration during the VOT. Many studies have confirmed that in patients with sepsis tissue saturation after flow restoration increases substantially slower, as compared to patients without infection and to healthy volunteers $[39,40]$. The 
maintenance of slowed down saturation increases after 24 hours of treatment is associated with poorer prognosis [39]. Furthermore, tissue saturation in septic shock is found to increase significantly quicker in survivors compared to non-survivors $[29,43]$.

Georger and colleagues. [44] used the measurement of tissue saturation in thenar muscles to evaluate the effects of noradrenaline on microcirculation in patients with septic shock. According to them, noradrenaline paradoxically not only increases tissue saturation but also accelerates its increases during reperfusion after the VOT. Based on the VOT, Masip and co-workers [45] have observed that the administration of activated drotrecogin alpha (activated $C$ protein generated using genetic engineering methods; at present withdrawn) has beneficial effects on tissue perfusion. The authors stress that VOT-based analysis of tissue perfusion in patients with septic shock allows to assess the risk of death with high sensitivity and specificity.

The clinical usefulness of VOT was also evaluated in patients at risk of haemorrhagic shock after trauma. It has been suggested that VOT performed in this group of patients enabled to detect an early stage of shock masked by compensation mechanisms [46]. During this stage the parameters determined routinely, such as arterial pressure, heart rate and lactate concentrations, are not deviated from normal values while increases in tissue saturation during reperfusion is significantly slower [46].

Moreover, the usefulness of VOT was assessed in end-stage heart failure patients whose baselines values of saturation in muscles were significantly lower compared to healthy volunteers. Dobutamine and levosimendan infusions were found to have beneficial effects on tissue saturation values recorded in these patients; and the improvement pertained to both baseline values and the rate of tissue saturation increases [47].

\section{SUMMARY}

NIRS is a technique providing new possibilities of evaluating oxygenation of the brain and of other organs. At present, its essential and clinically best-documented application is to prevent neurological complications and cognitive dysfunctions in patients undergoing cardiac and thoracic aorta surgeries. Baseline values of brain saturation can be an important parameter for predicting the prognosis in cardiac surgery. The benefits resulting from the use of brain saturation monitoring observed in to-date studies as well as possible use of tissue saturation measurements for evaluation of microcirculation indicate that tissue saturation can be considered a useful parameter to assess the patient status as well as prognosis in anaesthesia and intensive care. The important questions regarding the lowest safe values of brain saturation and the extent of its decreases, which indicates a significantly higher risk of complications, still await answers [11]. Nevertheless, the limitations of tissue oximetry interpretation do not blight the benefits of its monitoring, and rapid advances in NIRS technology should allow their quick elimination. Despite the lack of uniform monitoring procedures, it seems undeniable that brain saturation monitoring provides additional relevant information regarding the patient status, more so that other easy to use and reliable parameters are lacking. Moreover, thanks to this monitoring procedure, anaesthesiologists can undertake interventions aimed at preventing neurological complications, which also increases the safety of anaesthesia.

\section{ACKNOWLEDGEMENTS}

1. The authors declare no financial disclosure.

2. The authors declare no conflict of interest.

\section{References}

1. Jöbsis FF: Noninvasive, infrared monitoring of cerebral and myocardial oxygen sufficiency and circulatory parameters. Science 1977; 198: 1264-1267.

2. Murkin $J M$, Arango M: Near-infrared spectroscopy as an index of brain and tissue oxygenation. Br J Anaesth 2009; 103: i3-i13. doi: 10.1093/bja/aep299.

3. Nagdyman $N$, Fleck $T$, Schubert $S$ et al.: Comparison between cerebral tissue oxygenation index measured by near-infrared spectroscopy and venous jugular bulb saturation in children. Intensive Care Med 2005; 31: 846-850.

4. Yoshitani K, Kawaguchi M, Miura N et al.: Effect of hemoglobin concentration, skull thickness, and the area of the cerebrospinal fluid layer on near-infrared spectroscopy measurements. Anesthesiology 2007; 106: 458-462.

5. Zanatta P, Forti A: Effectiveness of NIRS to sample the frontal brain cortex in all cardiac surgery patients. Minerva Anestesiol 2011; 77: 1124-1125.

6. Casati A, Fanelli G, Pietropaoli P et al.: Continuous monitoring of cerebral oxygen saturation in elderly patients undergoing major abdominal surgery minimizes exposure to potential hypoxia. Anesth Analg 2005; 101: 740-747.

7. Slater JP, Guarino T, Stack J et al.: Cerebral oxygen desaturation predicts cognitive decline and longer hospital stay after cardiac surgery. Ann Thorac Surg 2009; 87: 36-44.

8. Olsson C, Thelin S: Regional cerebral saturation monitoring with near-infrared spectroscopy during selective antegrade cerebral perfusion: Diagnostic performance and relationship to postoperative stroke. J Thorac Cardiovasc Surg 2006; 131: 371-379.

9. Sorensen $\mathrm{H}$, Secher $\mathrm{NH}$, Siebenman $\mathrm{C}$ et al.: Cutaneous vasoconstriction affects near-infrared spectroscopy determined cerebral oxy gen saturation during administration of norepinephrine. Anesthesiology 2012; 117: 263-270.

10. Murkin JM: Is it better to shine a light, or rather to curse the darkness? Cerebral near-infrared spectroscopy and cardiac surgery. Eur J Cardio-Thorac Surg 2013; 43: 1081-1083. doi: 10.1093/ejcts/ezt186.

11. Grocott HP, Davie SN: Future uncertainties in the development of clinical cerebral oximetry. Front Physiol 2013; 4: 360. doi: 10.3389/fphys.2013.00360.

12. Vernick WJ, Gutsche JT: Pro: Cerebral oximetry should be a routine monitor during cardiac surgery. J Cardiothorac Vasc Anesth 2013; 27: 385-389.

13. Yao FF, Tseng CA, Ho CA, Levin SK, Illner P: Cerebral oxygen desaturation is associated with early postoperative neuropsychological dysfunction in patient undergoing cardiac surgery. J Cardiothorac Vase Anesth 2004; 18: 552-558. doi: 10.1053/j. jvca.2004.07.007.

14. Lassnigg A, Hiesmayr M, Keznicki P, Mullner T, Ehrlich M, Grubhofer G: Cerebral Oxygenation during cardiopulmonary bypass measured by near-infrared spectroscopy: Effects of 
hemodilution, temperature, and flow. J Cardiothorac Vasc Anesth 1999; 13: 544-548.

15. Deschamps A, Lambert J, Couture et al.: Reversal of decreases in cerebral saturation in high-risk cardiac surgery. J Cardiothorac Vasc Anesth 2013; 27: 1260-1266. doi: 10.1053/j. jvca2013.01.019.

16. Murkin JM, Adams SJ, Novick RJ et al.: Monitoring brain oxygen saturation during coronary bypass surgery: A randomized, prospective study. Anesth Analg 2007; 104: 51-58. doi: 10.1213/01ane0000246814.29362.f4.

17. Moerman A, Vandenplas G, Bove T, Wouters PF, De Hert SG: Relation between mixed venous oxygen saturation and cerebral oxygen saturation measured by absolute and relative near-infrared spectroscopy during off-pump coronary artery bypass grafting. $\mathrm{Br} J$ Anaesth 2013; 110: 258-265. doi: 10.1093/bja/aest375.

18. Paquet $C$, Deschamps $A$, Denault $A Y$ et al.: Baseline regional cerebral oxygen saturation correlates with left ventricular systolic and diastolic function. J Cardiothorac Vasc Anesth 2008; 22: 840-846. doi: 10.1053/j.jvca.2008.02.013.

19. Heringlake M., Garbers C, Käbler JH et al.: Preoperative cerebral oxygen saturation and clinical outcomes in cardiac surgery. Anesthesiology 2011; 114: 58-69. doi: 10.1097/ ALN.0b013e3181fef34e.

20. Zheng F, Sheinberg $R$, Yee MS, Ono M, Zheng Y, Hogue CW: Cerebral near-infrared spectroscopy (NIRS) monitoring and neurologic outcomes in adult cardiac surgery: A systematic review. Anesth Analg 2013; 116: 663-676. doi: 10.1213/ ANE.0b013e318277a255.

21. Kucewicz-Czech E, Urbańska E, Wolski P et al.: Monitorowanie regionalnej saturacji mózgowej podczas zabiegów kardiochirurgicznych - algorytm postępowania. Kardiochir Torakochir Pol 2011; 4: 504-508.

22. Moritz S, KasprzakP,Arlt M, Taeger K, Metz C: Accuracy of cerebral monitoring in detecting cerebral ischemia during carotid endarterectomy: a comparison of transcranial Doppler sonography, near-infrared spectroscopy, stump pressure, and somatosensory evoked potentials. Anesthesiology 2007; 107: 563-569.

23. Hirofumi O, Otone E, Hiroshil et al.:The effectiveness of regional cerebral oxygen saturation monitoring using near-infrared spectroscopy in carotid endarterectomy. J Clin Neurosci 2003; 10: 79-83. doi: 10.1016/S0967-5868(02)00268-0.

24. Carlin RE, McGraw DJ, Calimlim JR, Mascia ME: The use of near-infrared cerebral oximetry in awake carotid endarterectomy. J Clin Anesth 1998; 10: 109-113. doi: 10.1016/S09528180(97)00252-3.

25. Grubhofer G, PlöchI W, Skolka M, Czerny M, Ehrlich M, Lassnigg A: Comparing Doppler ultrasonography and cerebral oximetry as indicators for shunting in carotid endarterectomy. Anesth Analg 2009; 91: 1339-1344.

26. Mille T, Tachimiri ME, Klersy C et al.: Near infrared spectroscopy monitoring during carotid endarterectomy: which threshold value is critical? Eur J Vasc Endovasc Surg 2004; 27: 646-650.

27. Al-Rawi PG, Kirkpatrick PJ:Tissue oxygen index: thresholds for cerebral ischemia using near-infrared spectroscopy. Stroke 2006; 37: 2720-2725.

28. Kazan R, Bracco D, Hemmerling TM: Reduced cerebral oxygen saturation measured by absolute cerebral oximetry during thoracic surgery correlates with postoperative complication. Br J Anaesth 2009; 103: 811-816. doi: 10.1093/bja/aep.309.

29. Tang L, Kazan R, Taddei R, Zaouter C, Cyr S, Hemmerling TM: Reduced cerebral oxygen saturation during thoracic surgery predicts early postoperative cognitive dysfunction. $\mathrm{Br} J \mathrm{An}$ aesth 2012; 108: 623-629. doi: 10.1093/bja/aer501.

30. Casati A, Fanelli G, Pietropaoli P et al.: Monitoring cerebral oxygen saturation in elderly patients undergoing general abdominal surgery: a prospective cohort study. Eur J Anaesthesiol 2007; 24: 59-65.

31. Owens GE, King K, Gurney JG, Charpie JR: Low renal oximetry correlates with acute kidney injury after infant cardiac surgery. Pediatr Cardiol 2011;32: 183-188. doi: 10.1007/s00246-010-9839-x.

32. Weiss $M$, Schulz $G$, Teller l et al.: Tissue oxygenation monitoring during major pediatric surgery using transcutaneous liver near infrared spectroscopy. Paediatr Anaesth 2004; 14: 989-995.
33. Kim JW, Shin WJ, Park I, Chung IS, GwakM, Hwang GS: Splanchnic oxygen saturation immediately after weaning from cardiopulmonary bypass can predict early postoperative outcomes in children undergoing congenital heart surgery. Pediatr Cardiol 2014; 35: 587-595. doi: 10.1007/s00246-013-0824-z.

34. Fortune PM, WagstaffM, Petros AJ:Cerebro-splanchnic oxygenation ratio (CSOR) using near infrared spectroscopy may be able to predict splanchnic ischaemia in neonates. Intensive Care Med 2001; 27: 1041-1407. doi: 10.1007/s001340100994.

35. Gómez H, Torres A, Polanco P et al:: Use of non-invasive NIRS during a vascular occlusion test to assess dynamic tissue $\mathrm{O}_{2}$ saturation response. Intensive Care Med 2008; 34: 1600-1607. doi: 10.1007/s00134-008-1145-1.

36. Vallet $B$ : Vascular reactivity and tissue oxygenation. Intensive Care Med 1998; 24: 3-11.

37. Lima A, van Bommel J, Sikorska K et al.: The relation of nearinfrared spectroscopy with changes in peripheral circulation in critically ill patients. Crit Care Med 2011, 39: 1649-1654. doi: 10.1097/ccM.0b013e3182186675.

38. Lima A, van Bommel J, Jansen TC, Ince C, Bakker J: Low tissue oxygen saturation at the end of early goal-directed therapy is associated with worse outcome in critically ill patients. Crit Care 2009; 13 (Suppl 5): S13. doi: 10.1186/cc8011.

39. Creteur J, Carollo T, Soldati G, Buchele G, De Backer D, Vincent JL: The prognostic value of muscle $\mathrm{StO}_{2}$ in septic patients. Intensive Care Med 2007; 33: 1549-1556.

40. Skarda DE, Mulier KE, Myers DE, Taylor JH, Beilman GJ: Dynamic near-infrared spectroscopy measurements in patients with severe sepsis. Shock 2007; 27: 348-353.

41. De Blasi RA, Palmisani $S$, Mercieri $M$ et al.: Microvascular dysfunction and skeletal muscle oxygenation assessed by phasemodulation near-infrared spectroscopy in patients with septic shock. Intensive Care Med 2005; 31: 1661-1668.

42. Pareznik R, Knezevic R, Voga G, Podbregar M: Changes in muscle tissue oxygenation during stagnant ischemia in septic patients. Intensive Care Med 2006; 32: 87-92.

43. Saphiro NI, Arnold R, Sherwin R et al.: The association of nearinfrared spectroscopy derived tissue oxygenation measurements with sepsis syndromes, organ dysfunction and mortality in emergency department patients with sepsis. Crit Care 2011; 15: R223, doi: 10-1007/s11739-013-0973-3.

44. Georger JF1, Hamzaoui O, Chaari A, Maizel J, Richard C, Teboul JL: Restoring arterial pressure with norepinephrine improves muscle tissue oxygenation assessed by near-infrared spectroscopy in severely hypotensive septic patients. Intensive Care Med 2010; 36: 1882-1889. doi: 10.1007/s00134-010-2013-3.

45. Masip J, Mesquida J, Luengo Cet al.: Near-infrared spectroscopy $\mathrm{StO}_{2}$ monitoring to assess the therapeutic effect of drotrecogin alfa (activated) on microcirculation in patients with severe sepsis or septic shock. Ann Intensive Care 2013; 3: 30. doi: 10.1186/2110-5820-3-30.

46. Smith J, Bricker S, Putnam B: Tissue oxygen saturation predicts the need for early blood transfusion in trauma patients. Am Surg 2008; 74: 1006-1011.

47. Nanas S, Gerovasili V, Dimopoulos S et al.: Inotropic agents improve the peripheral microcirculation of patients with endstage chronic heart failure. J Card Fail 2008; 14: 400-406. doi: 10.1016/j.cardfail2008.02.001.

\section{Corresponding author:}

Aleksandra Biedrzycka, MD, PhD

Department of Anaesthesiology

and Intensive Therapy, University

Clinical Centre in Gdańsk

ul. Dębinki 7, 80-952 Gdańsk, Poland

e-mail:biedrzycka.ola@gmail.com

Received: 9.04.2015

Accepted: 29.07.2015 